\title{
High expression levels of DEF6 predicts a poor prognosis for patients with clear cell renal cell carcinoma
}

\author{
ZHEN-PENG ZHU ${ }^{1,2}$, LAN-RUO LIN ${ }^{3}$, TONG-DE LV ${ }^{1,2}$, \\ CHUN-RU XU ${ }^{1,2}$, TIAN-YU CAI ${ }^{1,2}$ and JIAN LIN ${ }^{1,2}$ \\ ${ }^{1}$ Department of Urology, Peking University First Hospital; ${ }^{2}$ Institute of Urology, Peking University, \\ Beijing 100034; ${ }^{3}$ College of Basic Medicine, Capital Medical University, Beijing 100069, P.R. China
}

Received February 25, 2020; Accepted July 1, 2020

DOI: $10.3892 /$ or.2020.7736

\begin{abstract}
Clear cell renal cell carcinoma (ccRCC) is one of the most common types of malignant tumors and early detection contributes to a better prognosis. Finding new biomarkers for the diagnosis or treatment remains meaningful. DEF6 guanine nucleotide exchange factor (DEF6) is upregulated in ccRCC compared to normal controls, but the relationship between DEF6 expression and prognosis in ccRCC is unclear. Moreover, the potential biological functions of DEF6 in ccRCC remains unclear. In the present study, the Cancer Genome Atlas (TCGA), Gene Expression Omnibus (GEO), TISIDB and the clinical database of the Peking University First Hospital were used to analyze DEF6 expression in ccRCC. Immunohistochemistry (IHC), western blotting and reverse transcription-quantitative PCR were used to examine the DEF6 protein and mRNA expression levels in cell lines and clinical samples. Subsequently, the Kaplan-Meier method and Cox regression analyses were used to determine the impact of DEF6 expression on the overall survival of patients alongside other clinical variables in both the TCGA database and the present clinical database. The results showed that both DEF6 mRNA and protein expression levels were upregulated in ccRCC compared to normal controls. The Kaplan-Meier survival analysis showed that patients with high DEF6 expression had poor prognoses from both the TCGA database and the present clinical database. Univariate survival analysis
\end{abstract}

Correspondence to: Dr Jian Lin, Department of Urology, Peking University First Hospital, 8 Xishiku street, Xicheng, Beijing 100034, P.R. China

E-mail: linjianbj@163.com

Abbreviations: ccRCC, clear cell renal cell carcinoma; DEGs, differentially expressed genes; GEF, guanine nucleotide exchange factor; GEO, Gene Expression Omnibus; GO, gene ontology; GSEA, gene set enrichment analysis; KEGG, Kyoto encyclopedia of genes and genomes; OS, overall survival; PPI, protein-protein interaction; RCC, renal cell carcinoma; TCGA, the Cancer Genome Atlas

Key words: DEF6 guanine nucleotide exchange factor, ccRCC, bioinformatics analyses, prognosis, immune microenvironment and multivariate survival analysis revealed that DEF6 could be an independent prognostic factor for ccRCC. Additionally, bioinformatics analysis indicated that differentially expressed genes related to DEF6 expression influenced ccRCC by regulating the tumor immune microenvironment. In conclusion, overexpression of DEF6 is significantly correlated with a poor prognosis for patients with ccRCC and DEF6 may influence the biological processes involved with ccRCC by regulating the immune microenvironment.

\section{Introduction}

Renal cell carcinoma (RCC) is one of the most common malignant tumor types worldwide, representing approximately $3-4 \%$ of all human cancers (1). The major histological type of RCC is clear cell RCC (ccRCC), which accounts for $>70 \%$ of all types of kidney cancers (2). The early detection of ccRCC contributes to a better prognosis (3). In the past decade, the genetic alterations behind ccRCC have been studied using bioinformatics analyses $(4,5)$. Bioinformatics analyses have become one of the most effective tools for analyzing human diseases (6). In addition, rapid technological advances led by academic institutions have continued to broaden the application of high-throughput sequencing technology from research to the clinic (7). Furthermore, scientists have proposed numerous potential genes that are related to the prognosis for patients with ccRCC. However, few genes have been discovered that are valid targets for diagnosis or treatment $(3,8)$. Therefore, finding further novel biomarkers of ccRCC, for the diagnosis or treatment, remains fruitful.

ccRCC is a highly immune-infiltrated tumor (9). Historically, ccRCC was one of the first malignant tumor types that responded to immunotherapy and continues to be among the most responsive $(10,11)$. Previous studies have shown that $\mathrm{T}$ cells are the most abundant immune-infiltrating cells in ccRCC (12). Hence, genes that regulate the functions of immune cells, especially $\mathrm{T}$ cells, may be correlated with the prognosis and effectiveness of immunotherapy for patients with ccRCC.

DEF6 guanine nucleotide exchange factor [GEF (DEF6)], is a 631 amino acid Rho-family GEF $(13,14)$. It is highly expressed in $\mathrm{T}$ and $\mathrm{B}$ cells, and regulates various immune-related processes such as the activation of $\mathrm{CD}^{+}$ 
$\mathrm{T}$ cells and the differentiation of $\mathrm{T}$ helper cells $(15,16)$. It also regulates cell morphology in cooperation with activated Rac1, and affects cell differentiation in collaboration with integrins $(17,18)$. More importantly, as a GEF, DEF6 can activate genes from the Rho-GTPase family, which contribute to tumor proliferation, migration and invasion (19). Previous studies have indicated that high expression levels of DEF6 predict a poor prognosis for colorectal cancer, ovarian carcinoma and breast cancer (20-22). It has also been demonstrated that there are high expression levels of DEF6 in RCC (23). Nevertheless, to the best of our knowledge, the relationship between DEF6 and ccRCC is unknown. Therefore, the present study explored the relationship between DEF6 expression and the prognosis for ccRCC.

To assess the relationship between the expression of DEF6 and its prognostic value and potential biological functions for patients with ccRCC, DEF6 was explored in the TCGA database, GEO database, TISIDB and the clinical database of Peking University First Hospital, Beijing, China. It was found that high DEF6 expression levels predicted a poor prognosis for patients with ccRCC. Furthermore, bioinformatics analyses revealed that DEF6 may regulate the components of the immune microenvironment to influence the processes behind ccRCC.

\section{Materials and methods}

Extraction of clinical and gene expression data from the ccRCC databases. Transcription profiles from high-throughput sequencing fragments per kilobase per million (HTSeq-FPKM) and corresponding clinical information were obtained from the TCGA website (https://www.cancer.gov/about-nci/organization/ccg/research/structural-genomics/tcga), which includes 539 ccRCC samples and 72 normal samples. Nine microarray datasets were also downloaded from the GEO website (https://www.ncbi.nlm.nih.gov/gds/). The data from the GEO databases were translated into $\log 2$ values for sequencing analysis. According to a previously reported method, if standardized data were not available, the raw data were downloaded. If a gene was found to have multiple probes in the same chip, the average value of all probes was taken as the expression value of the gene (24). Samples which were neither ccRCC, nor adjacent kidney tissue, were excluded from the present study. The 'sva' R package (version 3.6.1) was used to remove the batch effect (25). There were more than 700 specimens obtained from the following GEO datasets: GSE4282, GSE46699, GSE53757, GSE15641, GSE68417, GSE14994, GSE40435, GSE71963 and GSE76351.

Patients and specimens. A total of 146 paired samples from patients diagnosed with ccRCC were included in the resent study. The patients ranged in age from 20 to 83 years, including 97 males and 51 females. All patients underwent renal resection at Peking University First Hospital between June 2008 and January 2011. Clinical data of the recruited patients was obtained from medical records, such as Fuhrman score and body mass index (BMI). The present study was supported by the Ethics Committee of Peking University First Hospital and written informed consent was obtained from all patients. All procedures were performed according to the World Medical Association Declaration of Helsinki.

Immunohistochemistry (IHC). Firstly, the tissue samples were fixed in $10 \%$ formalin for $24 \mathrm{~h}$ at room temperature. Subsequently, the 4- $\mu \mathrm{m}$ paraffin-embedded tissue sections were prepared at room temperature. Immunostaining was performed using a two-step detection kit (cat. no. PV-9000; OriGene Technologies, Inc.) according to the manufacturer's protocol. The sections were deparaffinized in xylene at room temperature, rehydrated in a graded alcohol series and then boiled in citrate buffer ( $\mathrm{pH} \mathrm{6.0)}$ ) for $30 \mathrm{~min}$ in an autoclave. Endogenous peroxidases were blocked by incubation in $3 \%$ $\mathrm{H}_{2} \mathrm{O}_{2}$ for 30 min at room temperature. The sections were washed in PBS, blocked with $10 \%$ goat serum (OriGene Technologies, Inc.) at room temperature for $1 \mathrm{~h}$ and incubated with anti-DEF6 (cat. no. ab247011; 1:20,000; Abcam) at $4^{\circ} \mathrm{C}$ overnight. The sections were washed in PBS solution three times and incubated with a reaction enhancer kit (cat. no. PV-9000; OriGene Technologies, Inc.) for $20 \mathrm{~min}$ at room temperature, then washed in PBS solution three times. The sections were then incubated with peroxidase-conjugated secondary antibodies (cat. no. PV-9000; 1:1,000; OriGene Technologies, Inc.) for $30 \mathrm{~min}$ at room temperature. All slides were counterstained with DAB solution for $3 \mathrm{~min}$ and $20 \%$ hematoxylin for $1 \mathrm{~min}$ at room temperature and dehydrated. The primary antibody diluent was used as a negative control.

Evaluation of immunostaining staining. Two experienced independent investigators ( $\mathrm{ZZ}$ and $\mathrm{CX}$ ) examined all tumor slides by examining five random fields of view and observing 100 cells per view at $\mathrm{x} 400$ magnification using a light microscope (Olympus Corporation). The staining intensity was classified as 0 (no staining), 1 (weak), 2 (moderate) or 3 (strong); The proportion of stained tumor cells was scored as $0(0-5 \%)$, $1(6-25 \%), 2(26-50 \%), 3(51-75 \%), 4(>75 \%)$. The product of these two variables was used to calculate a final score based on a previous study (26), as follows: 0 (product of 0-3); 1 (product of 4-6); 2 (product of 7-9); 3 (product of 10-12).

Cell lines. HK-2, 293, 786-O, 769-P, Caki-1, ACHN, A498 and OSCR2 were acquired from the American Type Culture Collection. Cells were cultured in DMEM (Invitrogen; Thermo Fisher Scientific, Inc.) or 1640 media (Invitrogen; Thermo Fisher Scientific) containing 10\% FCS (Invitrogen; Thermo Fisher Scientific, Inc.) and $1 \%$ penicillin-streptomycin (Gibco; Thermo Fisher Scientific, Inc.). Cells were cultured in $10 \mathrm{~mm}$ culture dishes in a $5 \%$ humidified atmosphere at $37^{\circ} \mathrm{C}$.

Reverse transcription-quantitative PCR (RT-qPCR). Total RNA from 20 paired clinical samples was obtained from 20 patients diagnosed with ccRCC by Peking University First Hospital and extracted using TRIzol reagent ${ }^{\circledR}$ according to the manufacturer's protocol (Invitrogen; Thermo Fisher Scientific). Subsequently, cDNA was synthesized from 5-10 $\mu \mathrm{g}$ of total RNA using the Super Master Mix synthesis kit (Takara Bio, Inc), and the used condition of synthesizing cDNA were as follow: $15 \mathrm{~min}$ at $37^{\circ} \mathrm{C}, 5 \mathrm{sec}$ at $85^{\circ} \mathrm{C}$ and $5 \mathrm{~min}$ at $4^{\circ} \mathrm{C}$. Then, quantification of all gene transcripts was performed by RT-qPCR using the SYBR Premix ExTaq kit (Takara Bio, 
A
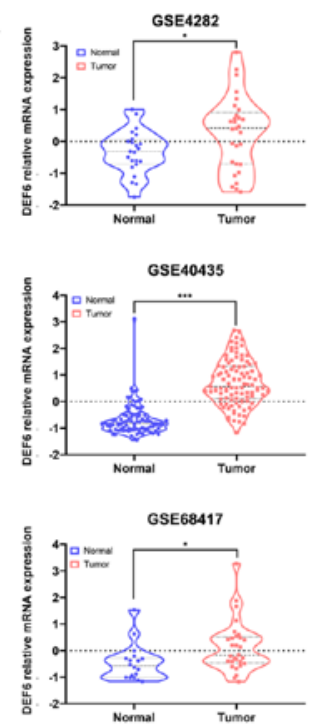
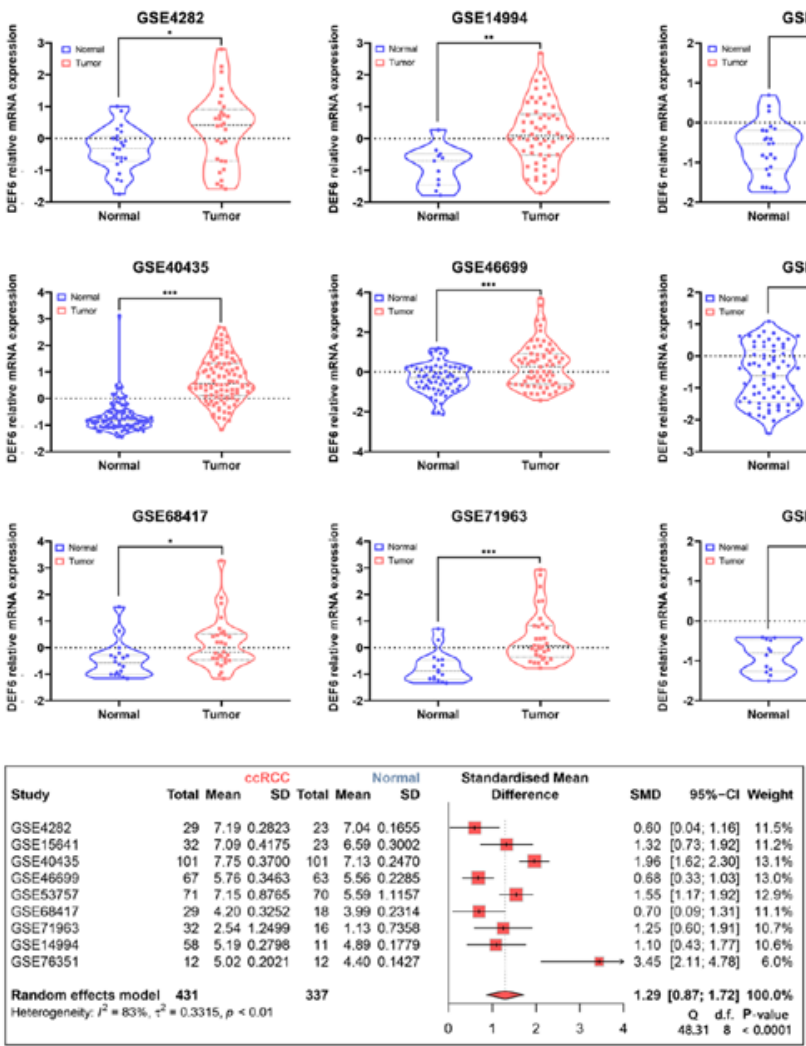
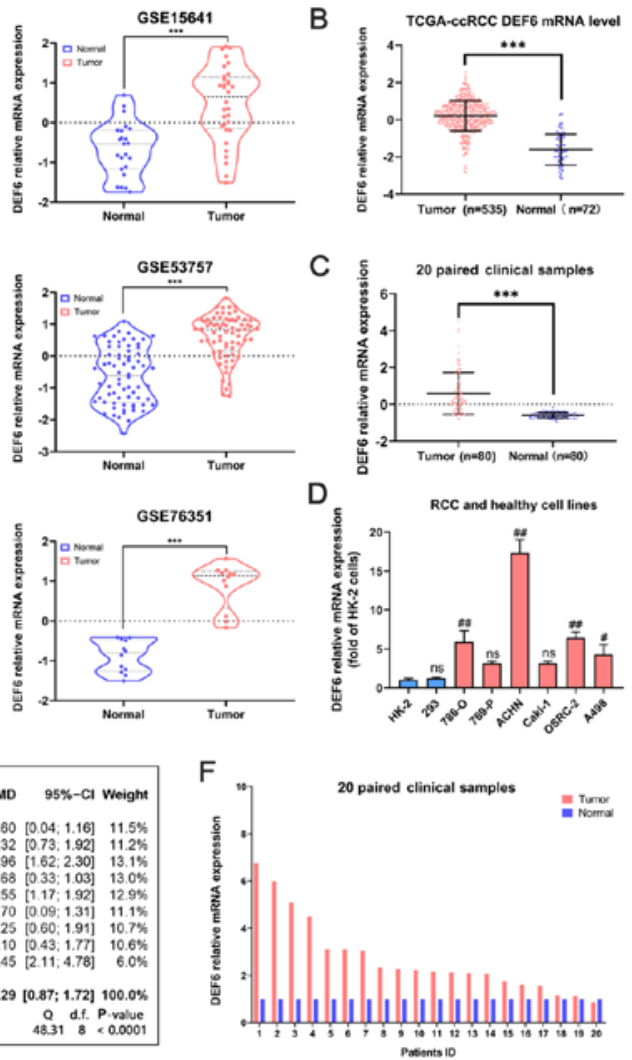

Figure 1. The DEF6 mRNA expression levels in ccRCC samples. (A) The mRNA expression levels of DEF6 in ccRCC tissues and adjacent normal renal tissues was compared. Nine databases were employed, namely, the GSE4282, GSE14994, GSE15641, GSE40435, GSE46699, GSE53757, GSE68417, GSE71963 and GSE76351 databases. The colored lines surrounding the data show the densities at the various expression levels in a violin chart. The mRNA expression levels of DEF6 in ccRCC tissues and adjacent normal renal tissues was compared in the (B) TCGA database and (C) 20 paired clinical samples. (D) The mRNA expression levels of DEF6 in various kidney cell lines was explored. (E) A forest plot of nine GEO databases was created. (F) The mRNA levels of 20 paired clinical samples are shown. ${ }^{*} \mathrm{P}<0.05,{ }^{* *} \mathrm{P}<0.01,{ }^{* * *} \mathrm{P}<0.001 .{ }^{*} \mathrm{P}<0.01,{ }^{\# \#} \mathrm{P}<0.001$ vs. HK-2 cells. ccRCC, clear cell RCC; CI, confidence interval; DEF6, DEF6 guanine nucleotide exchange factor; d.f., degrees of freedom; ns, not significant; RCC, renal cell carcinoma; SMD, standardized mean difference.

Inc.) and $\alpha$-tubulin was used as a normalizing control. The primer pairs used were as follows: DEF6 forward primer, 5'-TACATGCCCTACCTCAACAAGT-3' and reverse primer, 5'-TGTTCCCGTTGCTATCTGCC-3'; $\alpha$-tubulin forward primer, 5'-ACCTTAACCGCCTTATTAGCCA-3' and reverse primer, 5'-CACCACGGTACAACAGGCA-3'. Each reaction was performed four times and the qPCR conditions used were as follows: $10 \mathrm{~min}$ at $95^{\circ} \mathrm{C}, 40$ cycles of $15 \mathrm{sec}$ at $95^{\circ} \mathrm{C}$ and $1 \mathrm{~min}$ at $60^{\circ} \mathrm{C}$. The $2^{-\Delta \Delta \mathrm{Cq}}$ method was used to calculate the relative gene expression level (27). The relative mRNA expression levels were further normalized using the following formula: $\mathrm{Z}=(\mathrm{x}-\mu) / \sigma$, where ' $\mathrm{x}$ ' represents the observation of the sample expression, ' $\mu$ ' represents the sample mean expression, and ' $\sigma$ ' represents the sample SD (28).

Western blot analysis. Total proteins from cells were extracted using the NP-40 lysis buffer (cat. no. P0013F; Beyotime Institute of Biotechnology) and quantified using the BCA method. The supernatant (20 $\mu \mathrm{g}$ of protein) was denatured and separated on $10 \%$ SDS-PAGE. Samples were then transferred to $0.22-\mu \mathrm{m}$ PVDF membranes and blocked in skimmed milk for $1 \mathrm{~h}$ at room temperature. After that, samples were incubated overnight at $4{ }^{\circ} \mathrm{C}$ with the antibodies against DEF6 (cat. no. ab247011; 1:1,000; Abcam) and $\beta$-actin (cat. no. sc47778; 1:1,000; Santa Cruz Biotechnology, Inc.). After incubation with peroxidase-coupled anti-rabbit
IgG (cat. no. 7074; 1:1,000, Cell Signaling Technology, Inc.) at $37^{\circ} \mathrm{C}$ for $2 \mathrm{~h}$, bound proteins were visualized using an ECL kit (Pierce; Thermo Fisher Scientific, Inc.) and detected using a DNR Bioimaging System (DNR Bio-Imaging Systems, Ltd.). Relative protein levels were quantified using $\beta$-actin as the loading control.

Identification of differentially expressed genes (DEGs) related to DEF6 expression. According to the DEF6 median expression in ccRCC samples, the analyzed groups were divided into a low DEF6 expression group $(n=269)$ and a high DEF6 expression group ( $n=270)$ (29). Subsequently, the 'DESeq2' package and 'edgeR' $\mathrm{R}$ package (version 3.6.1) were used to screen DEGs between samples. Those with an absolute log2 fold-change $>1$ and a false discovery rate (FDR) $<0.05$ in the TCGA or GEO database were considered statistically significant.

Protein-protein interaction (PPI) network analysis. STRING version 11.0 (https://string-db.org/) was used to evaluate the PPI information of all DEGs related to DEF6 expression. The PPI network included 108 nodes and 324 edges. Subsequently, Cytoscape (version 3.7.1; http://cytoscape.org/) was used to analyze the PPI network, where a low degree value is correlated with a small node size, and a low co-expression value is related to a small edge size. Furthermore, genes with degrees $\geq 20$ 


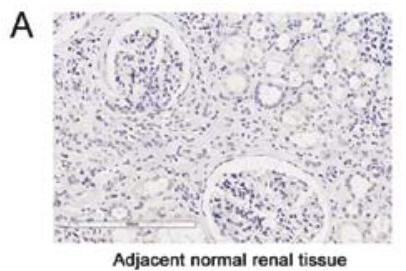

C

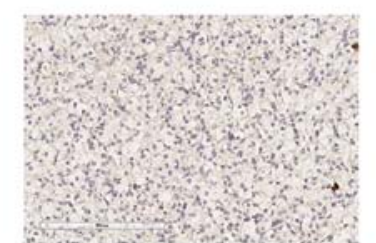

0,Negative

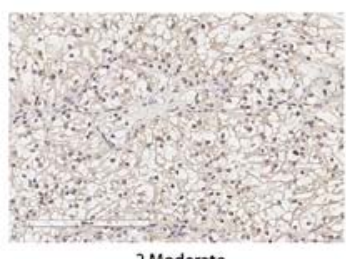

2,Moderate

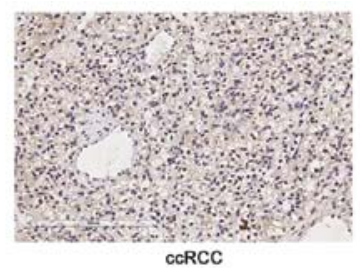

ccRCC

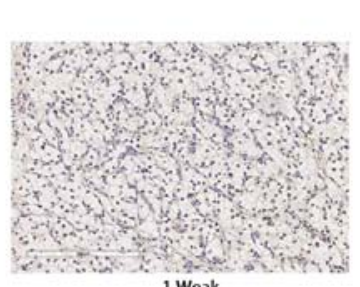

1, Weak

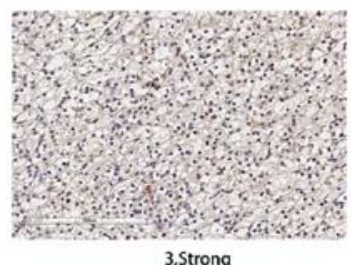

B

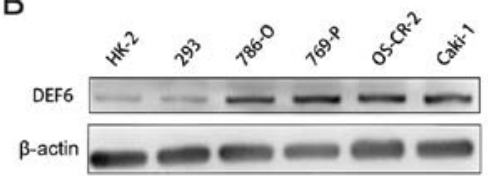

D
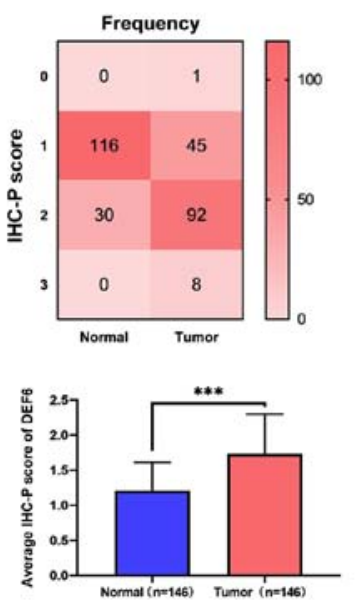

Figure 2. The DEF6 protein expression levels in ccRCC samples. (A) Representative positive DEF6 staining in adjacent normal renal tissue and ccRCC tissue. Scale bar, $200 \mu \mathrm{m}$. (B) Western blot analysis of DEF6 expression levels in ccRCC cell lines and normal cell lines. (C) Four representative images of the DEF6 expression profile as interpreted by IHC as negative, weak, moderate and strong. Scale bar, $200 \mu \mathrm{m}$. (D) Frequency distributions of DEF6 protein expression profiles across the cohort and the average scores from IHC. ${ }^{* * *} \mathrm{P}<0.001$. ccRCC, clear cell renal cell carcinoma; DEF6, DEF6 guanine nucleotide exchange factor; IHC, immunohistochemistry; IHC-P, immunohistochemistry-paraffin.

were selected as hub genes, and interactions (combined score $>0.4$ ) were considered significant. The interaction network of these proteins was visualized using Cytoscape 3.7.1 and the molecular complex detection (MCODE) plug selected necessary modules were applied (both MCODE score and node number $>4)(30)$.

Functional enrichment analyses of DEGs related to DEF6 expression. To further explore the mechanism of action DEF6 in regulating ccRCC, gene ontology (GO) enrichment analysis and Kyoto Encyclopedia of Genes and Genomes (KEGG) pathway analysis were performed using the 'ggplot2' $\mathrm{R}$ package (version 3.6.1; http://www.rstudio.com/). A P-value of $<0.05$ was considered statistically significant (31).

Moreover, gene set enrichment analysis (GSEA) was performed to identify the potential biological pathways. GSEA software (version 4.0.3; http://software.broadinstitute. org/gsea/index.jsp) was conducted on the JAVA 8.0 platform. By using the TCGA database, the high group and low group were classified according to the average mRNA expression levels of DEF6. For each analysis, gene set permutations were implemented 1,000 times. Significantly enriched gene sets were identified, which produced FDR q-values $<0.05$ (32).

TISIDB analysis. The TISIDB database (http://cis.hku. hk/TISIDB) integrated high-throughput data from 988 reported immune-related anti-tumor genes. The database enabled the analysis of correlations for the expression of selected genes with the expression of lymphocytes, immunomodulators and chemokines (33). In the present study, the relationships between the expression levels of DEF6 and lymphocytes as well as immunomodulators across various types human cancers, especially ccRCC, were investigated.

Statistical analysis. In the present study, unpaired t-tests were used to compare the mRNA expression levels in ccRCC tissues and normal renal tissues from the TCGA and GEO database, using SPSS 26.0 (IBM Corp.). One-way ANOVAs were conducted to calculate the difference among multiple groups, and Bonferroni's corrections were used for the post-hoc tests. Patients with unclear values (NX, MX, GX) or missing values were excluded from subsequent analyses. Paired t-tests were performed to calculate the differences between paired samples and the Wilcoxon signed rank test was used to calculate the differences among IHC scores of clinical samples. The Pearson's correlation analysis was used to calculate the correlation between DEF6 expression and clinical parameters. The Kaplan-Meier method and Cox regression were used to compare the impact of DEF6 expression on the overall survival (OS) of patients alongside other clinical variables in both the TCGA database and the present clinical database. All P-values are based on a two-sided statistical analysis, and $\mathrm{P}<0.05$ was considered to indicate a statistically significant difference.

\section{Results}

DEF6 mRNA expression levels are upregulated in ccRCC. the DEF6 mRNA expression levels were assessed in ccRCC using the GEO database, the TCGA database, clinical samples collected from the present study and cell lines. Firstly, compared with normal samples, in the ccRCC samples, the DEF6 mRNA expression levels were upregulated in the 9 GEO datasets 
A
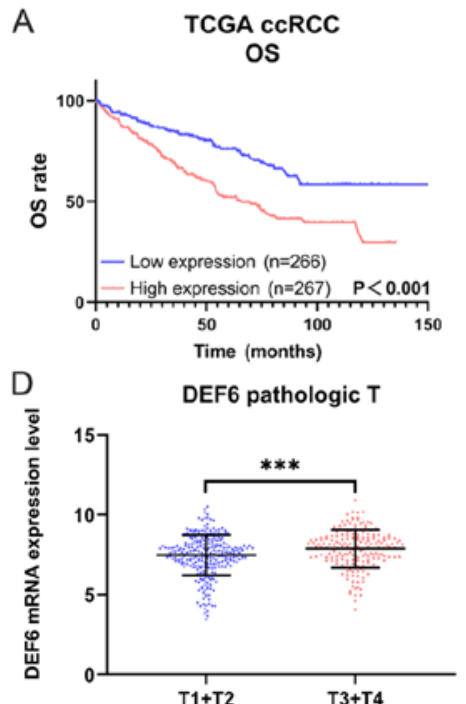

B
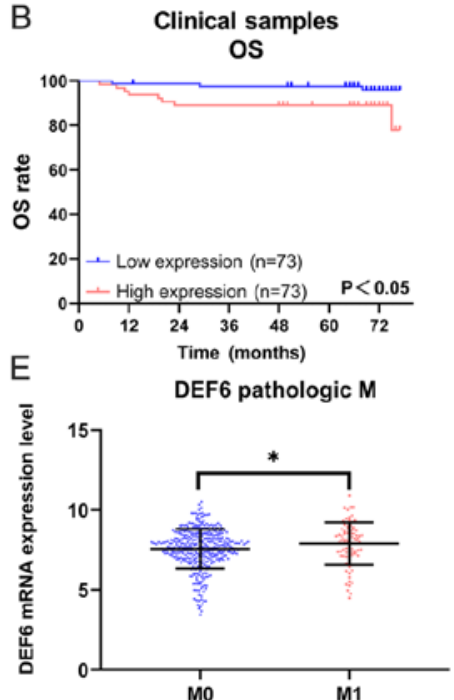

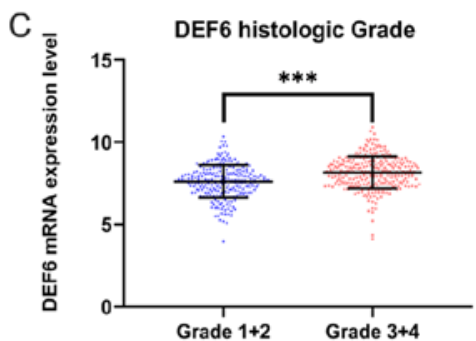

$\mathrm{F}$

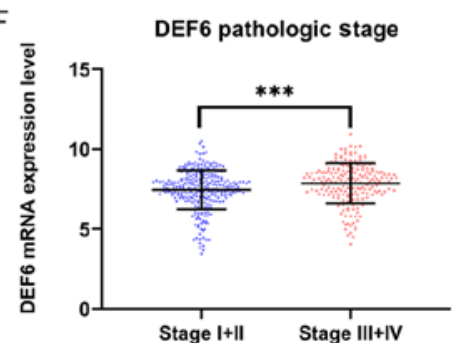

Figure 3. Kaplan-Meier survival curves for the OS of patients related to DEF6 in the (A) TCGA database and (B) the present clinical database. Furthermore, the correlation between DEF6 expression levels and clinicopathological characteristics in patients with ccRCC was explored in the TCGA database, including (C) histologic grade, (D) pathologic T stage, (E) pathologic M stage and (F) pathologic stage. ${ }^{*} \mathrm{P}<0.05,{ }^{* * * *} \mathrm{P}<0.001$. DEF6, DEF6 guanine nucleotide exchange factor; OS, overall survival.

(Fig. 1A). Furthermore, a forest plot, based on the standardized mean difference, showed the meta-analysis of 9 GEO datasets (Fig. 1E). The outcomes similarly indicated that DEF6 mRNA expression levels in ccRCC samples were upregulated in the 9 GEO datasets compared with the normal samples. In the TCGA database, the DEF6 mRNA expression levels were also increased in ccRCC (Fig. 1B). Furthermore, the DEF6 mRNA expression levels were elevated in 20 paired clinical samples (Fig. 1C). The relative DEF6 mRNA expression levels between the ccRCC tissues and paired normal tissues in each sample is also shown (Fig. 1F). In most samples, the mRNA expression levels of DEF6 in the ccRCC tissues was higher than that of the normal controls. In addition, the outcome of RT-qPCR in RCC cell lines showed that the DEF6 mRNA expression levels were significantly upregulated in the 786-O, ACHN, OSRC-2, and A498 cells compared to the HK-2 cells (Fig. 1D).

DEF6 protein expression levels are upregulated in ccRCC. Representative staining of tissues (Fig. 2A) and the representative scores (Fig. 2C) are shown. A higher DEF6 expression level was observed in the ccRCC tissue according to the IHC scores of 146 paired ccRCC tissues and corresponding adjacent normal renal tissues. The mean IHC scores of ccRCC tissues and adjacent normal renal tissues were 1.73 and 1.20, which showed significant difference. The frequency distribution of these scores is demonstrated in Fig. 2D. Western bolt analysis also showed that the DEF6 protein expression levels were increased in the ccRCC cell lines compared with the healthy cell lines (Fig. 2B).

Overexpression of DEF6 is an unfavorable prognostic factor for patients with $c c R C C$. To evaluate the prognostic value of DEF6 in ccRCC, the patient OS in both the TCGA database and the present study's clinical database were analyzed using the Kaplan-Meier log-rank test. It was found that high DEF6 expression was correlated with a poor prognosis for patients with ccRCC in both the TCGA database and the present database (Fig. 3A and B). Furthermore, the correlation between DEF6 expression and clinicopathological characteristics in patients with ccRCC was explored in the TCGA database. DEF6 expression was significantly associated with the pathologic grade (Fig. 3C), pathologic T (Fig. 3D), pathologic M (Fig. 3E) and pathologic stage (Fig. 3F).

Whether DEF6 could be an independent prognostic factor for patients with ccRCC was subsequently explored. Cox regression analysis was conducted using both the TCGA database and the present clinical database. Detailed patient information from both the TCGA database (Table SI) and the present clinical database (Table SII) is shown. As shown in Table SI, the clinical characteristics (size, pathologic T, pathologic $\mathrm{M}$ and pathologic $\mathrm{N}$ ) showed a significant difference between the high- and low-DEF6 expression groups in the TCGA database. Meanwhile, as shown in Table SII, the clinical characteristics (size) showed a significant difference between the high- and low-DEF6 expression groups in the present clinical database.

In the TCGA database, univariate Cox regression analysis showed that DEF6 expression, age at diagnosis, tumor size, histologic grade, pathologic $\mathrm{T}$ and pathologic $\mathrm{M}$ stages were correlated with the OS of patients with ccRCC (Table I). Moreover, multivariate Cox regression analysis indicated that DEF6 expression, age at diagnosis and pathologic $M$ stage were independent prognostic factors for OS (Table I).

Moreover, in the present clinical database, DEF6 expression, tumor size and pathologic $\mathrm{T}$ were correlated with the OS of patients with ccRCC (Table II). In addition, multivariate Cox regression analysis indicated that DEF6 expression and pathologic T stage were independent prognostic factors for OS (Table II).

Biological analysis of DEF6 in ccRCC. The analysis contained 539 ccRCC samples in the TCGA database, 101 ccRCC 
Table I. Univariate analysis and multivariate analysis of overall survival in the TCGA database.

\begin{tabular}{lccccc}
\hline & \multicolumn{2}{c}{ Univariate analysis } & & \multicolumn{2}{c}{ Multivariate analysis } \\
\cline { 2 - 3 } Parameters & HR $(95 \% \mathrm{CI})$ & P-value & & HR $(95 \%$ CI $)$ & P-value \\
\hline DEF6 level, high vs. low & $2.076(1.502,2.869)$ & $<0.001$ & & $1.856(1.335,2.580)$ & $<0.001$ \\
Age at initial pathologic diagnosis, $>60$ vs. $<60$ years & $1.734(1.248,2.410)$ & $<0.01$ & & $1.593(1.142,2.222)$ & $<0.01$ \\
Gender, male vs. female & $1.077(0.776,1.496)$ & 0.657 & & \\
Size, longest dimension, $\geq 2$ cm vs. $<2$ cm & $1.526(1.114,2.091)$ & $<0.01$ & & \\
Histologic grade, G1 + G2 vs. G3 + G4 & $2.332(1.640,3.315)$ & $<0.001$ & & \\
Pathologic T, T3 + T4 vs. T1 + T2 & $2.739(2.003,3.747)$ & $<0.001$ & & \\
Pathologic N, N1 vs. N0 & $0.983(0.722,1.338)$ & 0.912 & & \\
Pathologic M, M1 vs. M0 & $4.289(3.126,5.884)$ & $<0.001$ & & $2.673(1.819,3.928)$ & $<0.001$ \\
\hline
\end{tabular}

CI, confidence interval; DEF6, DEF6 guanine nucleotide exchange factor; HR, hazard ratio.

Table II. Univariate analysis and multivariate analysis of overall survival in the clinical database of the present study.

\begin{tabular}{lccccr}
\hline & \multicolumn{2}{c}{ Univariate analysis } & & \multicolumn{2}{c}{ Multivariate analysis } \\
\cline { 2 - 3 } Parameters & HR $(95 \% \mathrm{CI})$ & P-value & & HR $(95 \% \mathrm{CI})$ & P-value \\
\hline DEF6 level, high vs. low & $7.981(1.058,60.211)$ & $<0.05$ & & $1.856(1.335,2.580)$ & $<0.05$ \\
Age at initial pathologic diagnosis, $\geq 60$ vs. $<60$ years & $1.898(0.721,4.966)$ & 0.194 & & \\
Gender, male vs. female & $1.334(0.468,3.801)$ & 0.590 & & \\
Size, longest dimension, $\geq 6$ cm vs. $<6 \mathrm{~cm}$ & $6.060(2.132,17.219)$ & $<0.01$ & & $<0.01$ \\
BMI, $\geq 23.9$ vs. $<23.9$ & $0.844(0.325,2.187)$ & 0.726 & & \\
Pathologic T, T3 + T4 vs. T1 + T2 & $10.859(3.977,29.647)$ & $<0.001$ & & $7.101(1.965,25.659)$ & $<$ \\
Fuhrman score, 2+3 vs. 1 & $4.898(0.649,36.992)$ & 0.123 & & \\
\hline
\end{tabular}

BMI, body mass index; CI, confidence interval; DEF6, DEF6 guanine nucleotide exchange factor; HR, hazard ratio.

samples in GSE40435 and 71 ccRCC samples in GSE53757. A total of 188 overlapping DEGs related to DEF6 expression were identified from 3 datasets, including 180 upregulated genes and 8 downregulated genes (Fig. 4A).

Moreover, GO, KEGG and GSEA analyses were conducted to explore the biological functions of these DEGs. The GO analysis showed that the DEGs were mainly enriched in the activation and regulation of the immune microenvironment (Fig. 4C). Moreover, the KEGG pathway enrichment analysis indicated that the primary functions of the identified DEGs were the regulation of the immune microenvironment. Interestingly, programmed cell death ligand 1 expression and programmed cell death 1 checkpoint pathways in cancer were also enriched in the KEGG pathway (Fig. 4D). Furthermore, GSEA analysis showed that 7 gene sets were upregulated and 4 gene sets were downregulated, with significant enrichment at both the NOM P-value $<0.05$ and FDR q-value $<0.05$ (Table III). These gene sets were mainly correlated with the immune response and tumor metabolism (Fig. 4B).

Construction of the PPI network. To identify the hub genes of these DEGs related to DEF6 expression, a PPI network was constructed using STRING 11.0 (https://string-db.org/), including 108 nodes and 324 edges. Subsequently, Cytoscape (version 3.7.1) was used to analyze the PPI network, where a low degree value is correlated with a small node size and a low co-expression value is related to a small edge size. The co-expression network showed that protein tyrosine phosphatase receptor type c (PTPRC), integrin subunit beta 2 (ITGB2), lymphocyte-specific protein tyrosine kinase (LCK) and cytotoxic T-lymphocyte associated protein 4 (CTLA4) were hub genes with a degree $>20$ (Fig. 5A). The MCODE plugin was then used to explore the critical modules of target genes and two imperative modules were identified with MCODE score and node number $>4$ (Fig. 5B and C). Two imperative modules might act as the core regulatory network for the biological functions.

Regulation of molecules involved with the immune microenvironment by DEF6. The aforementioned biological analyses showed that DEF6 was associated with the immune microenvironment. As such, the TISIDB database was used to explore the correlations between DEF6 expression and lymphocytes and immunomodulators, using Spearman's correlation tests. 
Table III. The enrichment of GSEA gene sets at both the NOM P-value $<0.05$ and FDR q-value $<0.05$.

\begin{tabular}{lrrrrc}
\hline GS follow link to MSigDB & Size & ES & NES & NOM P-value & FDR q-value \\
\hline HALLMARK_ALLOGRAFT_REJECTION & 199 & 0.81 & 2.32 & $<0.001$ & 0.007 \\
HALLMARK_INTERFERON_GAMMA_RESPONSE & 198 & 0.75 & 2.2 & 0.002 & 0.016 \\
HALLMARK_INFLAMMATORY_RESPONSE & 198 & 0.7 & 2.15 & $<0.001$ & 0.017 \\
HALLMARK_IL6_JAK_STAT3_SIGNALING & 86 & 0.73 & 2.14 & $<0.001$ & 0.014 \\
HALLMARK_INTERFERON_ALPHA_RESPONSE & 95 & 0.74 & 2.06 & 0.009 & 0.021 \\
HALLMARK_IL2_STAT5_SIGNALING & 196 & 0.56 & 2 & 0.004 & 0.032 \\
HALLMARK_COMPLEMENT & 199 & 0.56 & 1.95 & 0.017 & 0.038 \\
HALLMARK_FATTY_ACID_METABOLISM & 157 & -0.57 & -2.07 & 0.014 & 0.037 \\
HALLMARK_OXIDATIVE_PHOSPHORYLATION & 184 & -0.68 & -2.06 & 0.017 & 0.027 \\
HALLMARK_ADIPOGENESIS & 193 & -0.5 & -1.94 & 0.022 & 0.045 \\
HALLMARK_ANDROGEN_RESPONSE & 96 & -0.55 & -1.94 & 0.02 & 0.037
\end{tabular}

ES, enrichment score; FDR, false discovery rate; NES, normalized enrichment score; GS, gene sets; NOM, normalized.

A

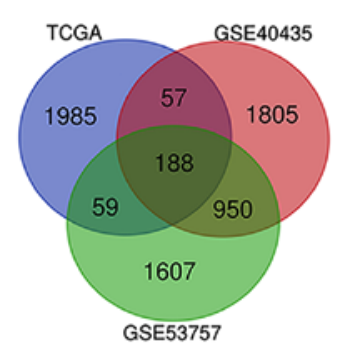

B

C

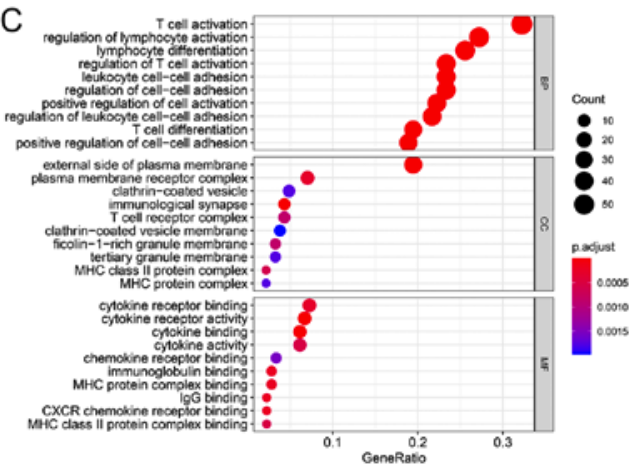

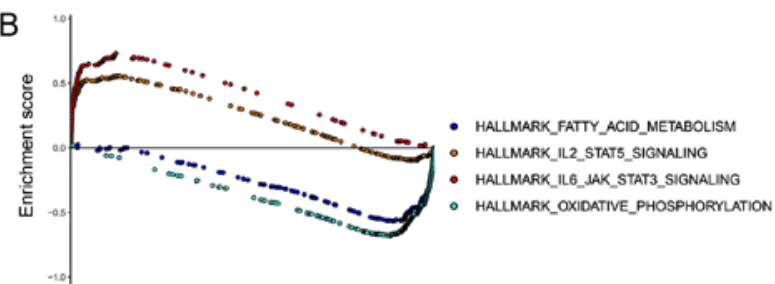

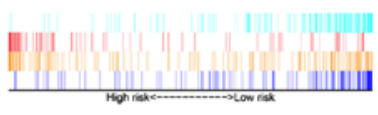

D

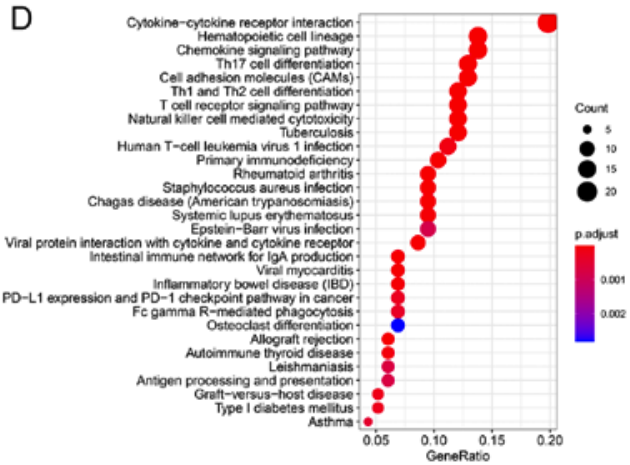

Figure 4. (A) The 188 overlapping DEGs from three datasets: TCGA, GSE40435 and GSE53757. (B) Representative GSEA results showing enrichment of the immune response and tumor metabolism in ccRCC is shown. The dot plot images of (C) GO and (D) KEGG were constructed, where larger dot sizes are correlated with higher counts and a darker red color is related to lower P-value. CAM, cell adhesion molecule; CXCR, chemokine receptor type 4; DEG, differentially expressed genes; IBD, inflammatory bowel disorder; Ig, immunoglobulin; MHC, major histocompatibility complex; PD-1, programmed cell death 1; PD-L1, programmed cell death ligand 1; Th, T helper cell.

Figs. S1A and 6A showed the correlation between DEF6 expression and tumor-infiltrating lymphocytes, and the 4 types of tumor-infiltrating lymphocytes that significant correlated with DEF6 expression, including myeloid-derived suppressor cells (MDSCs), activate CD8 T cells (Act_CD8), activate B cells (Act_B) and effector memory CD8 T cells (Tem_CD8). Moreover, Figs. S1B and 6B showed the correlation between DEF6 expression and immunoinhibitors, and the 4 significant immunoinhibitors included lymphocyte activating 3 (LAG3), programmed cell death 1 (PDCD1), T Cell Immunoreceptor
With Ig And ITIM Domains (TIGIT) and CD96. Furthermore, Figs. S1C and 6C showed the correlation between DEF6 expression and immunostimulators, and the 4 significant immunostimulators included CD27, Lymphotoxin Alpha (LTA), Killer Cell Lectin Like Receptor K1 (KLRK1) and CD48. Figs. S1D and 6D showed the correlation between DEF6 expression and major histocompatibility complexes (MHC) molecules, and the 4 significant MHC molecules included 4 major histocompatibility complexes (HLAs), which are HLA-DOB, HLA-DPB1, HLA-DMA and HLA-DRA. 

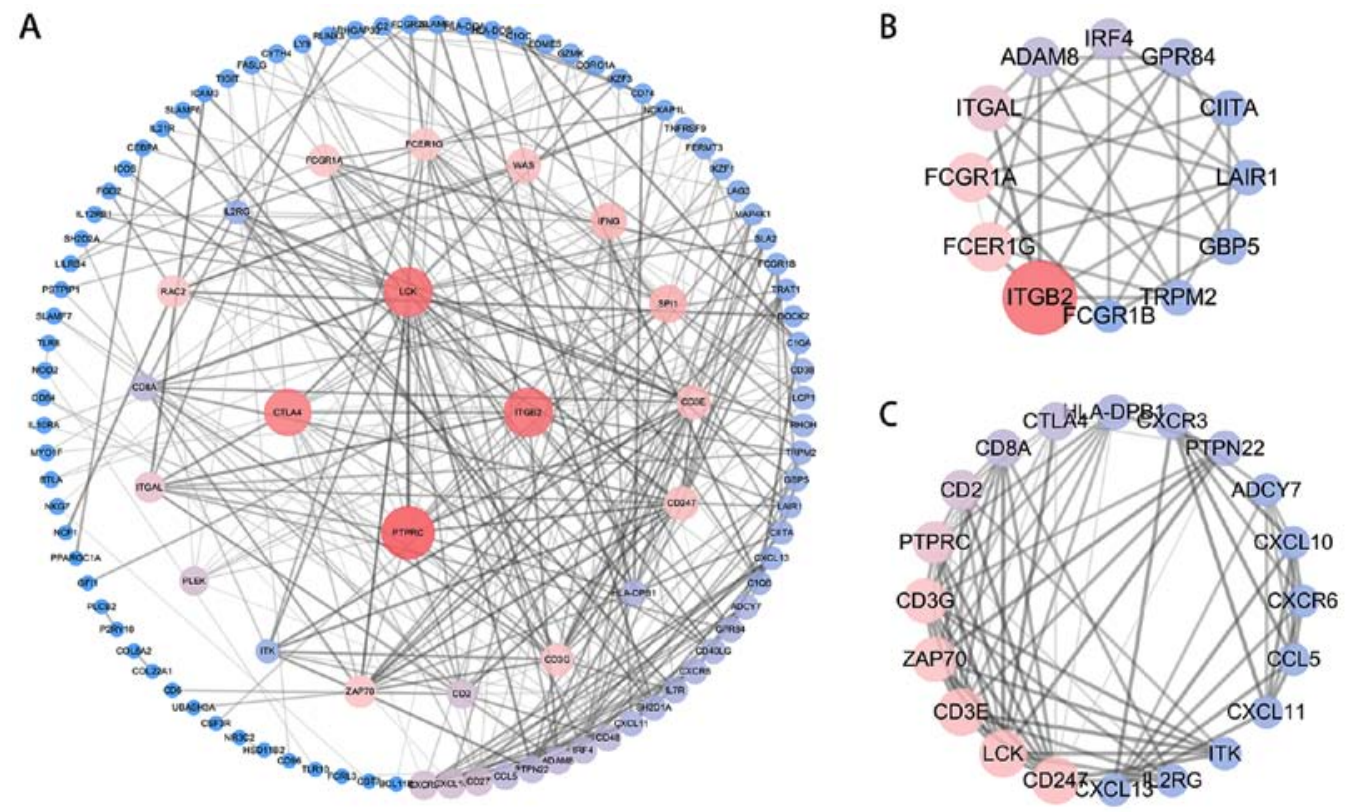

Figure 5. (A) A protein-protein interaction network was constructed and 4 hub genes with degree $>20$ were identified, where a low degree is correlated with a small node size and a low co-expression value is related to a small edge size. The bluer the color, the smaller the degree and the redder the color, the higher the degree. The degree refers to the number of connections the node has with other nodes. Selected important modules of the target gene with (B) MCODE score $\geq 4$ and $(C)$ nodes $\geq 4$. MCODE, molecular complex detection.
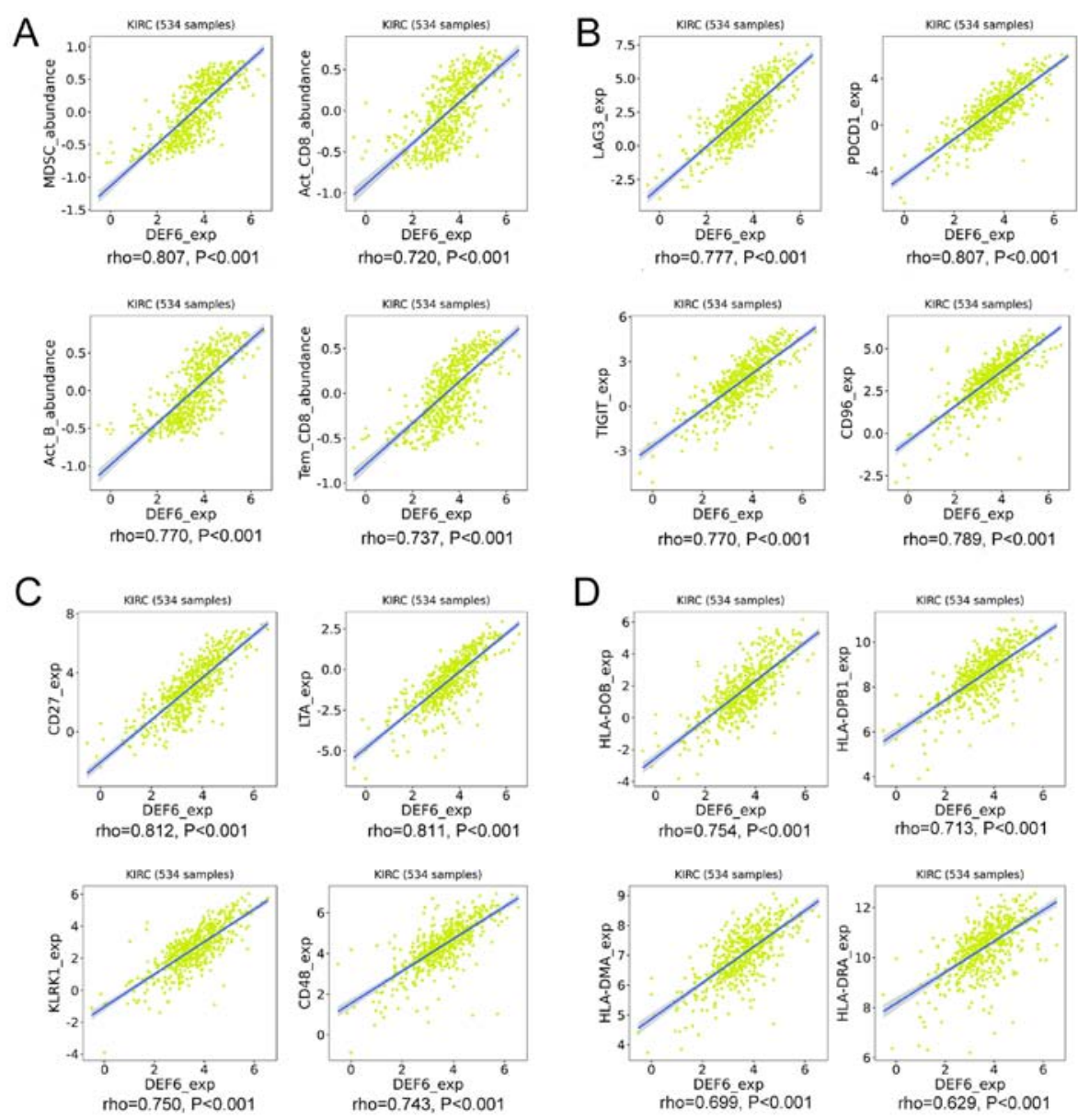

Figure 6. Spearman's correlation of expression of DEF6 with expression of 4 most significant lymphocytes and immunomodulators in the TISIDB database. (A) Correlations between DEF6 and the top 4 significant lymphocytes expression levels. (B) Correlations between DEF6 and the top 4 significant immunoinhibitors expression levels. (C) Correlations between DEF6 and the top 4 significant immunostimulators expression levels. (D) Correlations between DEF6 and the top 4 significant MHC molecules expression levels. Red and blue cells indicate positive and negative correlations, respectively. The color intensity is directly proportional to the strength of the correlations. DEF6, guanine nucleotide exchange factor; MHC, major histocompatibility complex. KIRC, kidney renal clear cell carcinoma. 
Hence, DEF6 may influence the immune microenvironment by regulating the aforementioned immune molecules.

\section{Discussion}

DEF6, also known as IBP or SWAP-70, is a GEF that regulates various processes associated with the immune microenvironment. GEFs are members of the diffuse B-cell lymphoma protein family and play a significant role in regulating the activation status of Rho-GTPases $(34,35)$. To the best of our knowledge, Rho-GTPases are associated with oncogenic activities and contribute to the processes of malignant tumor phenotypes such as migration, invasion and metastasis (36-38). Interestingly, as an upstream activator of the Rho-GTPase family, DEF6 is involved in various cellular processes, such as the cell polarity, microtubule dynamics, membrane transport pathways and transcription factor activity (39-41). Previous studies have demonstrated that overexpression of DEF6 contributes to a poor prognosis in various cancer types, such as ovarian carcinoma and prostate cancer $(42,43)$. However, the correlation between DEF6 expression and the prognosis in ccRCC is unclear.

A previous study showed the potential functions of DEF6 in regulating kidney podocytes (44). This study was the first to reveal the correlation between DEF6 expression and the prognosis in ccRCC. In the present study, both DEF6 mRNA and protein expression levels were explored in ccRCC. Compared with the adjacent normal tissue, the DEF6 mRNA expression level was upregulated in the TCGA database, GEO database and the present clinical samples. Moreover, 146 paired ccRCC tissues and their adjacent normal tissues were analyzed using IHC. Raised DEF6 expression levels were found in ccRCC. Univariate and multivariate Cox regression suggested that high DEF6 expression was significantly related to a poor prognosis for patients with ccRCC, which indicated that DEF6 may be an independent prognostic factor for ccRCC.

According to the DEF6 expression levels, the ccRCC samples were divided into the high expression group and low expression group. Subsequently, 'DESeq2' and 'edgeR' were used to analyze the transcription profile from the TCGA and GEO databases. A total of 188 DEGs were found for subsequent analysis, including 180 upregulated genes and 8 downregulated genes. The biological functions of these DEGs were explored using GO, KEGG and GSEA enrichment analyses.

The enrichment of biological processes indicated that DEF6 mainly regulated immune microenvironment molecules by influencing their activation, difference and adhesion. In addition, cell components showed that DEGs were enriched in various membranes such as the external side of plasma membrane and ficolin-1-rich granule membrane. These DEGs were also enriched in the composition of various complexes such as the MHC II complex and reporter complex, which suggested that DEF6 may influence the transmission of biological information. Moreover, molecular function analysis showed that DEF6 chiefly regulates cytokines and bind with various structures, such as immunoglobulins and the MHC II protein complex. Moreover, KEGG pathway analysis also indicated that DEF6 participates in various processes of the immune microenvironment. Interestingly DEF6 mainly influ- enced the functions of immune molecules, such as Th17 cell differentiation and natural killer cell mediated cytotoxicity, which indicated that DEF6 may regulate the development of ccRCC by influencing the immune microenvironment. In addition, GSEA analysis found 7 upregulated gene sets and 4 downregulated gene sets. Enrichment analyses of these gene sets showed that DEF6 was mainly involved in the immune response and tumor metabolism. Immune response-related signaling pathways, such as the interleukin (IL)2/STAT5 pathway and IL6/JAK/ STAT3 pathway, were upregulated. In addition, tumor metabolism-related signaling pathways, such as fatty acid metabolism and oxidative phosphorylation, were downregulated.

To explore the hub genes among DEGs, a PPI network was constructed and found 4 hub genes: PTPRC, ITGB2, LCK and CTLA4. The detailed information of the 4 hub genes was described as follows: PTPRC belongs to a member of the protein tyrosine phosphatase (PTP) family. PTPs are signaling molecules that regulate a variety of cellular processes, including cell growth, differentiation, mitosis and oncogenic transformation (45). PTPRC is an essential regulator of $\mathrm{T}$ cell and $\mathrm{B}$ cell antigen receptor signaling, which also suppresses JAK kinases (46). Moreover, ITGB2 is a crucial regulator of lymphocyte trafficking, activation and residence time (47). LCK is a member of the Src family and regulates the activation of $\mathrm{T}$ cells (48). Recent studies have shown that LCK is expressed in various tumor types, such as breast cancer, colon cancer and lung carcinoma (49-51). In addition, CLTA-4 is a member of the immunoglobulin superfamily and mediates opposing functions in $\mathrm{T}$ cell activation. These functions contribute to tumur development (52). A total of 4 hub genes were found to be involved in regulating the immune microenvironment. In addition, ITGB 2 and CLTA- 4 were also associated with various tumur processes.

The analyses performed in the present study showed that DEF6 is associated with the immune microenvironment. To the best of our knowledge, RCC has a high level of immune infiltration and $\mathrm{T}$ cells are the main immune cell type enriched in ccRCC $(53,54)$. As such, the TISIDB database was used to explore the correlation DEF6 expression with lymphocytes and immunomodulators. The results showed that DEF6 had the most significant correlation with lymphocytes (such as MDSCs, Act_CD8, Act_B and Tem_CD8), immunoinhibitors [such as LAG3, PDCD1, TIGIT and CD96], immunostimulators (such as CD27, LTA, KLRK1 and CD48), and MHC molecules (such as HLA-DOB, HLA-DPB1, HLA-DMA and HLA-DRA). $\mathrm{CD}^{+} \mathrm{T}$ cells have been shown to be associated with improved clinical outcomes and responses to immunotherapy (55). Moreover, TIGIT and CD96 are correlated with anti-tumor immunity (56). Combined with the outcome of the GSEA and KEGG analyses, TISIDB indicated that patients with ccRCC with high DEF6 expression may benefit more from immunotherapy.

There are several limitations to this study. Firstly, the biological functions of DEF6 in ccRCC cancer cell lines need to be verified in vitro. Moreover, the specific mechanisms of action by which DEF6 influences ccRCC remain unclear and require further study. In conclusion, the present study demonstrated for the first time that both DEF6 mRNA and protein levels were upregulated in ccRCC. Overexpression of DEF6 is 
an unfavorable prognostic factor for patients with ccRCC. In addition, the mechanism of action by which DEF6 regulates ccRCC may be associated with the immune microenvironment.

\section{Acknowledgements}

Sincere thanks is given for the experimental guidance from Dr Anbang He, Dr Cong Huang and Dr Guangjie Ji. Dr Anbang He and Dr Guangjie Ji gave us guidance on bioinformatics analyses. Moreover, Dr Cong Huang gave us guidance on cell culture and laboratory techniques.

\section{Funding}

This study was supported by the National Natural Science Foundation of China (grant no. 81670617).

\section{Availability of data and materials}

The datasets used and/or analyzed during the current study are available from the corresponding authors on reasonable request.

\section{Authors' contributions}

JL and ZPZ designed the study. JL obtained the financial support for this study. ZPZ collected the sequencing data from public datasets and analyzed the data. LRL, TYC and CRX collected the clinical samples and performed IHC staining, western blot experiments as well as RT-qPCR. ZPZ and TYC drafted and revised the manuscript. ZPZ and TDL performed bioinformatics analyses for this study. All authors have read and approved the final manuscript.

\section{Ethics approval and consent to participate}

For the use of the clinical materials for research purposes, the study was conducted following the Declaration of Helsinki. This study was conducted with the approval of the Peking University First Hospital Ethical Committee and informed consent was obtained from all participants.

\section{Patient consent for publication}

Not applicable.

\section{Competing interests}

The authors declare that they have no competing interests.

\section{References}

1. Siegel RL, Miller KD and Jemal A: Cancer statistics, 2020. CA Cancer J Clin 70: 7-30, 2020.

2. Nicholson HE, Tariq Z, Housden BE, Jennings RB, Stransky LA, Perrimon N, Signoretti S and Kaelin WG Jr: HIF-independent synthetic lethality between CDK4/6 inhibition and VHL loss across species. Sci Signal 12: 12, 2019.

3. Li QK, Pavlovich CP, Zhang H, Kinsinger CR and Chan DW: Challenges and opportunities in the proteomic characterization of clear cell renal cell carcinoma (ccRCC): A critical step towards the personalized care of renal cancers. Semin Cancer Biol 55: $8-15,2019$.
4. Cancer Genome Atlas Research Network: Comprehensive molecular characterization of clear cell renal cell carcinoma. Nature 499: 43-49, 2013.

5. Sato Y, Yoshizato T, Shiraishi Y, Maekawa S, Okuno Y,Kamura T, Shimamura T, Sato-Otsubo A, Nagae G, Suzuki H, et al: Integrated molecular analysis of clear-cell renal cell carcinoma. Nat Genet 45: 860-867, 2013.

6. Oliver GR, Hart SN and Klee EW: Bioinformatics for clinical next generation sequencing. Clin Chem 61: 124-135, 2015.

7. Xuan J, Yu Y, Qing T, Guo L and Shi L: Next-generation sequencing in the clinic: Promises and challenges. Cancer Lett 340: 284-295, 2013.

8. Majer W, Kluzek K, Bluyssen H and Wesoły J: Potential Approaches and Recent Advances in Biomarker Discovery in Clear-Cell Renal Cell Carcinoma. J Cancer 6: 1105-1113, 2015.

9. Yoshihara K, Shahmoradgoli M, Martínez E, Vegesna R, Kim H, Torres-Garcia W, Treviño V, Shen H, Laird PW, Levine DA, et al: Inferring tumour purity and stromal and immune cell admixture from expression data. Nat Commun 4: 2612, 2013.

10. Atkins MB, Regan M and McDermott D: Update on the role of interleukin 2 and other cytokines in the treatment of patients with stage IV renal carcinoma. Clin Cancer Res 10: 6342S-6346S, 2004.

11. Escudier B: Emerging immunotherapies for renal cell carcinoma. Ann Oncol 23 (Suppl 8): viii35-viii40, 2012.

12. Şenbabaoğlu Y, Gejman RS, Winer AG, Liu M, Van Allen EM, de Velasco G, Miao D, Ostrovnaya I, Drill E, Luna A, et al: Tumor immune microenvironment characterization in clear cell renal cell carcinoma identifies prognostic and immunotherapeutically relevant messenger RNA signatures. Genome Biol 17: 231, 2016.

13. Gupta S, Lee A, Hu C, Fanzo J, Goldberg I, Cattoretti G and Pernis AB: Molecular cloning of IBP, a SWAP-70 homologous GEF, which is highly expressed in the immune system. Hum Immunol 64: 389-401, 2003.

14. Mavrakis KJ, McKinlay KJ, Jones P and Sablitzky F: DEF6, a novel PH-DH-like domain protein, is an upstream activator of the Rho GTPases Rac1, Cdc42, and RhoA. Exp Cell Res 294: 335-344, 2004

15. Tanaka Y, Bi K, Kitamura R, Hong S, Altman Y, Matsumoto A, Tabata H, Lebedeva S, Bushway PJ and Altman A: SWAP-70-like adapter of $\mathrm{T}$ cells, an adapter protein that regulates early TCR-initiated signaling in Th2 lineage cells. Immunity 18: 403-414, 2003.

16. Canonigo-Balancio AJ, Fos C, Prod'homme T, Bécart S and Altman A: SLAT/Def6 plays a critical role in the development of Th17 cell-mediated experimental autoimmune encephalomyelitis. J Immunol 183: 7259-7267, 2009.

17. Oka T, Ihara S and Fukui Y: Cooperation of DEF6 with activated Rac in regulating cell morphology. J Biol Chem 282: 2011-2018, 2007.

18. Samson T, Will C, Knoblauch A, Sharek L, von der Mark K, Burridge $\mathrm{K}$ and Wixler V: Def-6, a guanine nucleotide exchange factor for Rac1, interacts with the skeletal muscle integrin chain alpha7A and influences myoblast differentiation. J Biol Chem 282: 15730-15742, 2007.

19. Lazer G and Katzav S: Guanine nucleotide exchange factors for RhoGTPases: Good therapeutic targets for cancer therapy? Cell Signal 23: 969-979, 2011.

20. Chen S, Han Q, Wang X, Yang M, Zhang Z, Li P, Chen A, Hu C and Li S: IBP-mediated suppression of autophagy promotes growth and metastasis of breast cancer cells via activating mTORC2/Akt/FOXO3a signaling pathway. Cell Death Dis 4: e842, 2013.

21. Xu Y, Hou Y, Liu T and Lou G: Overexpression and clinical significance of IBP in epithelial ovarian carcinoma. Oncol Lett 15: 6604-6610, 2018.

22. Zhang Z, Wang Q, Li P, Zhou Y, Li S, Yi W, Chen A, Kong P and $\mathrm{Hu}$ C: Overexpression of the Interferon regulatory factor 4-binding protein in human colorectal cancer and its clinical significance. Cancer Epidemiol 33: 130-136, 2009.

23. Otsubo T, Hida Y, Ohga N, Sato H, Kai T, Matsuki Y, Takasu H, Akiyama K, Maishi N, Kawamoto T, et al: Identification of novel targets for antiangiogenic therapy by comparing the gene expressions of tumor and normal endothelial cells. Cancer Sci 105: 560-567, 2014.

24. Liao Y, Yin G, Wang X, Zhong P, Fan X and Huang C: Identification of candidate genes associated with the pathogenesis of small cell lung cancer via integrated bioinformatics analysis. Oncol Lett 18: 3723-3733, 2019. 
25. Leek JT, Johnson WE, Parker HS, Jaffe AE and Storey JD: The sva package for removing batch effects and other unwanted variation in high-throughput experiments. Bioinformatics 28: 882-883, 2012

26. Zhu Z, He A, Lv T, Xu C, Lin L and Lin J: Overexpression of $\mathrm{P} 4 \mathrm{HB}$ is correlated with poor prognosis in human clear cell renal cell carcinoma. Cancer Biomark 26: 431-439, 2019.

27. Livak KJ and Schmittgen TD: Analysis of relative gene expression data using real-time quantitative PCR and the 2(-Delta Delta C(T)) Method. Methods 25: 402-408, 2001

28. Martinez-Millana A, Hulst JM, Boon M, Witters P, Fernandez-Llatas C, Asseiceira I, Calvo-Lerma J, Basagoiti I, Traver V, De Boeck K, et al: Optimisation of children z-score calculation based on new statistical techniques. PLoS One 13 e0208362, 2018

29. Lv W, Yu X, Li W, Feng N, Feng T, Wang Y, Lin H and Qian B Low expression of LINC00982 and PRDM16 is associated with altered gene expression, damaged pathways and poor survival in lung adenocarcinoma. Oncol Rep 40: 2698-2709, 2018.

30. Shannon P, Markiel A, Ozier O, Baliga NS, Wang JT, Ramage D, Amin N, Schwikowski B and Ideker T: Cytoscape: A software environment for integrated models of biomolecular interaction networks. Genome Res 13: 2498-2504, 2003.

31. Yu G, Wang LG, Han Y and He QY: clusterProfiler: An R package for comparing biological themes among gene clusters. OMICS 16: 284-287, 2012.

32. Subramanian A, Tamayo $P$, Mootha VK, Mukherjee S, Ebert BL, Gillette MA, Paulovich A, Pomeroy SL, Golub TR LanderES, et al: Gene setenrichment analysis: A knowledge-based approach for interpreting genome-wide expression profiles. Proc Natl Acad Sci USA 102: 15545-15550, 2005

33. Ru B, Wong CN, Tong Y, Zhong JY, Zhong SSW, Wu WC, Chu KC, Wong CY, Lau CY, Chen I, et al: TISIDB: An integrated repository portal for tumor-immune system interactions. Bioinformatics 35: 4200-4202, 2019.

34. Zheng Y: Dbl family guanine nucleotide exchange factors Trends Biochem Sci 26: 724-732, 2001.

35. Cook DR, Rossman KL and Der CJ: Rho guanine nucleotide exchange factors: Regulators of Rho GTPase activity in development and disease. Oncogene 33: 4021-4035, 2014.

36. Vega FM and Ridley AJ: Rho GTPases in cancer cell biology. FEBS Lett 582: 2093-2101, 2008.

37. Jansen S, Gosens R, Wieland T and Schmidt M: Paving the Rho in cancer metastasis: Rho GTPases and beyond. Pharmacol Ther 183: 1-21, 2018.

38. Li H, Peyrollier K, Kilic G and Brakebusch C: Rho GTPases and cancer. Biofactors 40: 226-235, 2014.

39. Etienne-Manneville S and Hall A: Rho GTPases in cell biology. Nature 420: 629-635, 2002.

40. Hall A: Rho family GTPases. Biochem Soc Trans 40: 1378-1382, 2012.

41. Jaffe AB and Hall A: Rho GTPases: Biochemistry and biology. Annu Rev Cell Dev Biol 21: 247-269, 2005.

42. Chiyomaru T, Tatarano S, Kawakami K, Enokida H, Yoshino H, Nohata N, Fuse M, Seki N and Nakagawa M: SWAP70, actin-binding protein, function as an oncogene targeting tumor-suppressive miR-145 in prostate cancer. Prostate 71 $1559-1567,2011$
43. Liew PL, Fang CY, Lee YC, Lee YC, Chen CL and Chu JS: DEF6 expression in ovarian carcinoma correlates with poor patient survival. Diagn Pathol 11: 68, 2016.

44. Worthmann K, Leitges M, Teng B, Sestu M, Tossidou I, Samson T, Haller H, Huber TB and Schiffer M: Def-6, a novel regulator of small GTPases in podocytes, acts downstream of atypical protein kinase C (aPKC) $\lambda /$. Am J Pathol 183: 1945-1959, 2013.

45. Lazo JS, McQueeney KE, Burnett JC, Wipf P and Sharlow ER: Small molecule targeting of PTPs in cancer. Int J Biochem Cell Biol 96: 171-181, 2018.

46. Porcu M, Kleppe M, Gianfelici V, Geerdens E, De Keersmaecker K, Tartaglia M, Foà R, Soulier J, Cauwelier B, Uyttebroeck A, et al: Mutation of the receptor tyrosine phosphatase PTPRC (CD45) in T-cell acute lymphoblastic leukemia. Blood 119: 4476-4479, 2012.

47. Reichardt P, Patzak I, Jones K, Etemire E, Gunzer M and Hogg N: A role for LFA-1 in delaying T-lymphocyte egress from lymph nodes. EMBO J 32: 829-843, 2013.

48. Stirnweiss A, Hartig R, Gieseler S, Lindquist JA, Reichardt P, Philipsen L, Simeoni L, Poltorak M, Merten C, Zuschratter $\mathrm{W}$, et al: $\mathrm{T}$ cell activation results in conformational changes in the Src family kinase Lck to induce its activation. Sci Signal 6: ra13, 2013 .

49. Clarke CN, Lee MS, Wei W, Manyam G, Jiang ZQ, Lu Y, Morris J, Broom B, Menter D, Vilar-Sanchez E, et al: Proteomic Features of Colorectal Cancer Identify Tumor Subtypes Independent of Oncogenic Mutations and Independently Predict Relapse-Free Survival. Ann Surg Oncol 24: 4051-4058, 2017.

50. Chakraborty G, Rangaswami H, Jain S and Kundu GC: Hypoxia regulates cross-talk between Syk and Lck leading to breast cancer progression and angiogenesis. J Biol Chem 281: 11322-11331, 2006.

51. Mahabeleshwar GH and Kundu GC: Tyrosine kinase p56lck regulates cell motility and nuclear factor kappaB-mediated secretion of urokinase type plasminogen activator through tyrosine phosphorylation of IkappaBalpha following hypoxia/reoxygenation. J Biol Chem 278: 52598-52612, 2003.

52. Rowshanravan B, Halliday N and Sansom DM: CTLA-4: A moving target in immunotherapy. Blood 131: 58-67, 2018.

53. Poprach A, Lakomý R and Büchler T: Immunotherapy of Renal Cell Carcinoma. Klin Onkol 30 (Suppl 3): 55-61, 2017 (In Czech).

54. Geissler K, Fornara P, Lautenschläger C, Holzhausen HJ, Seliger B and Riemann D: Immune signature of tumor infiltrating immune cells in renal cancer. OncoImmunology 4: e985082, 2015.

55. Tumeh PC, Harview CL, Yearley JH, Shintaku IP, Taylor EJ, Robert L, Chmielowski B, Spasic M, Henry G, Ciobanu V, et al: PD-1 blockade induces responses by inhibiting adaptive immune resistance. Nature 515: 568-571, 2014.

56. Zhang Q, Bi J, Zheng X, Chen Y, Wang H, Wu W, Wang Z, Wu Q, Peng H, Wei H, et al: Blockade of the checkpoint receptor TIGIT prevents NK cell exhaustion and elicits potent anti-tumor immunity. Nat Immunol 19: 723-732, 2018. International (CC BY-NC-ND 4.0) License. 